\title{
A 60-Year-Male Post Corneal Transplantation with Acute Pneumonia
}

\author{
Chamanant Satjanon1, Theerasuk Kawamatawong2 \\ ${ }^{1}$ Department of Medicine, Faculty of Medicine Ramathibodi Hospital, Mahidol University, Bangkok, Thailand \\ ${ }^{2}$ Division of Pulmonary and Critical Care Medicine, Department of Medicine, Faculty of Medicine Ramathibodi Hospital, \\ Mahidol University, Bangkok, Thailand \\ Email: may.ninthnov@gmail.com
}

How to cite this paper: Satjanon, C. and Kawamatawong, T. (2021) A-60-Year-Male Post Corneal Transplantation with Acute Pneumonia. Open Journal of Ophthalmology, 11, 18-24.

https://doi.org/10.4236/ojoph.2021.111002

Received: December 15, 2020

Accepted: February 2, 2021

Published: February 5, 2021

Copyright (c) 2021 by author(s) and Scientific Research Publishing Inc. This work is licensed under the Creative Commons Attribution International License (CC BY 4.0).

http://creativecommons.org/licenses/by/4.0/

\begin{abstract}
Pneumonia is a common complication in organ transplantation patients. Multiple respiratory pathogens such as bacteria, viruses and fungi are potentially coexisted. A 60-year-old male with left eye post corneal transplantation developed acute severe pneumonia caused by Pneumocystis jiroveci (PJP) coinfection with Nocardia spp. and Cytomegalovirus (CMV). He was hospitalized due to acute respiratory failure. Chest radiographs and chest Computed Tomography (CT) revealed extensive ground-glass opacities. PJP was diagnosed from Bronchoalveolar Lavage Fluid (BALF). The pneumonia was persistent despite of receiving intravenous cotrimoxazole. Tracheal aspirate showed faint gram-positive filamentous beaded branching organisms. Consequently Nocardia spp. was proven. Intravenous cotrimoxazole was continued and intravenous imipenem was added. After a course of dual antibiotics, pneumonia was gradually improved. A week after, he developed the worsened acute respiratory failure. The bronchoscopy was performed. The new pathogens were not detected from BALF microbiology. The BALF cytology was unremarkable. PJP was detected by Polymerase Chain Reaction (PCR) from BALF. CMV antigenemia was detected from BALF and blood. Intravenous ganciclovir was given. This report describes PJP coinfected with Nocardia spp. and CMV in post corneal transplantation patient suffering from severe pneumonia. Multiple respiratory pathogens are common among transplantation patients representing host immunosuppression and inadequate antimicorbial prophylaxis.
\end{abstract}

\section{Keywords}

Pneumocystis jiroveci Pneumonia, Nocardiosis., Cytomegalovirus (CMV), Corneal Transplantation, Acute Respiratory Failure, Glucocorticoid, Ground Glass Opacities, Brochoalveolar Lavage Fluid (BALF) 


\section{Introduction}

Respiratory complications are highly prevalent in immunocompromised patients including organ transplantation patients. Both infections and non-infectious processes have to be considered in pulmonary diseases related to transplantation. Polymicrobial infections are potentially detected in up to a third of transplanted patient presenting with pneumonia [1]. The diagnosis and treatment of pneumonia in these patients are challenging. Transplanted patients who have received immunosuppressive agents are susceptible to multiple opportunistic respiratory infections according to the degree of immunity defect and the provided prophylaxis regimes [2] [3].

\section{Case Report}

A 60-year-old male had left corneal transplantation 4 years prior to the current admission. He presented to an emergency department with high-grade fever and progressive shortness of breath for 3 days. The low-grade fever with dry cough and myalgia were also noted for a week. His left corneal graft was maintained only by methylprednisolone eye drop until 2 months ago. He went to the previous hospital due to progressive visual impairment in his left eye. He was diagnosed with late left corneal graft rejection and treated with oral high dose prednisolone $1 \mathrm{mg} / \mathrm{kg} /$ day for 2 weeks before tapering off. He still took $40 \mathrm{mg}$ of prednisolone at the time of presentation. The topical methylprednisolone eye drop was continued throughout. Cotrimoxazole and acyclovir were not given as prophylaxis.

At the initial presentation, he was febrile with a temperature of 38.9 Celsius. His blood pressure was $96 / 50 \mathrm{mmHg}$ and pulse rate was 123 beats per minute. Tachypnea with severe oxygen desaturation were detected during ambient air. He was intubated and mechalically ventilated. Physical examination revealed coarse crepitation at both lower lungs. Chest radiograph showed bilateral pulmonary opacity. Oral prednisolone was replaced by intravenous hydrocortisone due to hypotension which was suspected from sepsis or Critical Illness-Related Corticosteroid Insufficiency (CIRCI) Cortisol level was not measured at the time due to continuous treatment with prednisolone. Intravenous empirical antibiotics containing levofloxacin and ceftazidime were given for presumptive bacterial pneumonia. Gram stain of tracheal aspirate revealed no organism. Pulmonary opacities and hypoxemia were, however, progressed even after the treatment. Pneumocystis jiroveci Pneumonia (PJP) was then clinically suspected. Intravenous cotrimoxazole and cefepime were given, and intravenous levofloxacin and ceftazidime were discontinued. His symptoms became worsened at 24 hours after initiating treatments. Tracheal aspirate were microbiologically evaluated using gram strain, aerobic culture, and PCR for PJP. A chest Computed Tomography (CT) scan was also performed.

Chest CT scan (Figure 1) revealed basal lung predominantly located Ground Glass Opacities (GGO). The focal cavity lesion and internal hypodensity and air 
bronchogram were detected. His tracheal suction (Figure 2) gram strain showed faint gram-positive filamentous beaded branching organism. Pneumocystis jiroveci coinfected with Nocardia spp. causing severe pneumonia was confired. Intravenous cotrimoxazole and imipenem were given, while cefipime was discontinued. After a week of new treatment, his clinical symptoms were gradually improved. The aerobe culture of tracheal aspirate was later reported as Nocardia spp. After extubation, he developed dyspnea with unremarkable chest radiographic findings. Chest CT scan was again performed (Figure 3). Bronchoscopy was performed and Bronchoalveolar Lavage Fluid (BALF) was obtained. BALF stainings was unremarkable. Indirect Immunofluorescent Assay (IFA) for PJP was negative. However, PJP was remained detected by PCR technique. BALF culture was positive for Nocardia spp. Cytomegalovirus (CMV) viral load in BALF was 55,000 copies/ml while blood viral load was 689 copies/ml. Hence, probable CMV pneumonitis was diagnosis. Intravenous ganciclovir was given. His symptom was improved and the chest radiographic resolution of pneumonia was noted. He was extubated 10 days after the treatment with ganciclovir. The completely normal chest radiography was noted at the 6 months appointment (Figure 4). Oral trimethoprime and ganciclovir were prescribed as antimicrobial prophylaxis and regularly maintained.

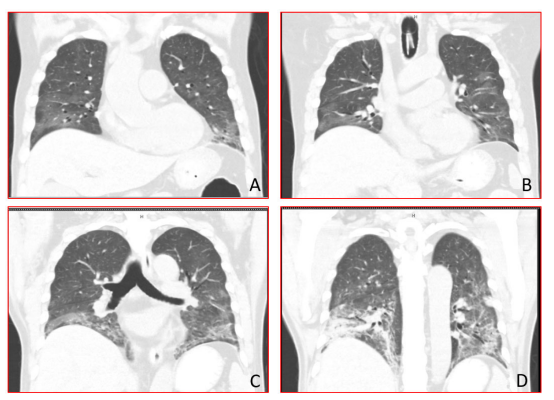

Figure 1. Show CT abnormalities of lung parenchyma during initial hospitalization. PJP and Nocardiosis were diagnosed by bronchoscopy. (A) and (B) show bilateral GGO occupying both basal lungs. (C) and (D) show reticular opacities with architectural distortion of both lower lung zones. Abbreviations. GGO; Groud-glass opacities, PJP; Pneumocystis jiroveci pneumonia.
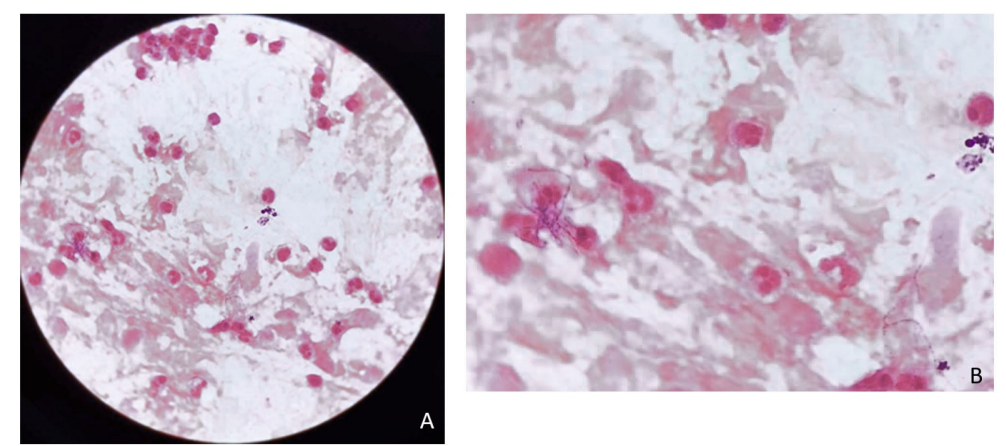

Figure 2. Gram strain of tracheal secretion showing numerous polymorphonuclear cells with gram positive staining organism, under $100 \times$ microscope (A). Higher magnification view showing gram positive beaded filamentous branching organism (B). 


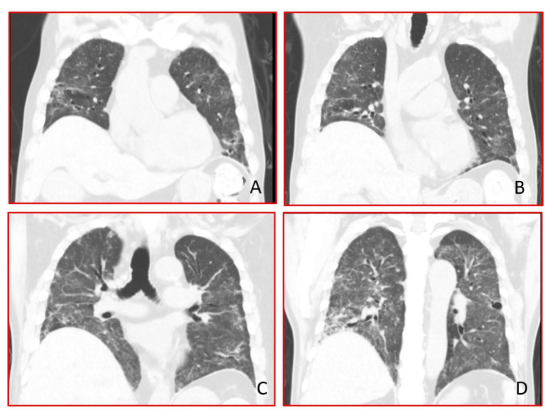

Figure 3. Show CT abnormalities of lung parenchyma after $14^{\text {th }}$ day SMX-TMP treatment. (A)-(C) show progressive reticulation with nodularity in both lungs; (D) shows extensive GGO and reticular opacity. Cystic changed and traction bronchiectasis were also noted. Abbreviation. SMX-TMP: Sulfamethoxazole-Trimethoprime

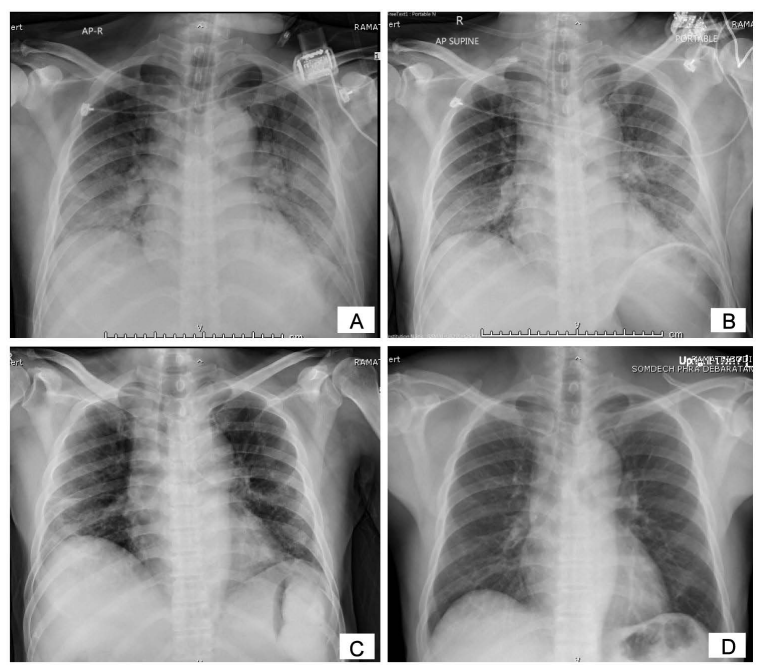

Figure 4. Chest radiography on the first day show patchy opacity predominate at both lower lungs zone with air bronchogram, (A). After PJP, Nocardia, and CMV pneumonia was diagnosed and continued treatment (B). At discharge date chest radiograph shows focal patchy opacity remains at right lower lung zone (C). After 6 months follow up chest radiography shows complete resolution of pneumonia (D).

\section{Discussion}

Most patients who underwent either solid organ or hematopoietic stem cell transplantation and received systemic immunosuppressive drugs were defined as immunocompromised patients. The different immunosuppressive agents carry risks for different infectious organisms due to distinct immunologic mechanisms. Antimicrobial prophylaxis is recommended in selected patients. Patients who received corticosteroids equivalent of prednisolone more than $20 \mathrm{mg}$ per day for at least 2 weeks were recommended for PJP prophylaxis [4]. Clinical manifestations of Pneumocystis jiroveci pneumonia are non-specific and different between HIV and non-HIV group. In HIV-seronegative immunocompromised group, acute onset of symptoms including fever, nonproductive cough, shortness of breath, and hypoxia are common [4] [5]. Compared to HIV group, classic triads of exertional dyspnea, dry cough, and fever might be seen and developed more sub- 
acutely [5]. Bilateral GGO and consolidation pattern are common chest radiograph findings in both groups. Chest CT typically shows widespread GGO and consolidations [5]. The clinical worsening of PJP after receiving definite treatment was observed in our patient. Since trimethoprime resistant PJP due to Dihydrofolate Reductase (DHFR) mutation has been occasionally reported and is associated with failure prophylaxis [6]. The worsening of PJP despite of trimethoprime indicating sub-therapeutic dose of trimethoprime and the cocomittent opportunistic infection such as viruses, nocardiosis, tuberculosis and other invasive fungi. Nocardiosis is common opportunistic infection in immunocompromised patient using corticosteroid including solid organ transplantation. The common clinical manifestations are fever, cough, and cutaneous abscess. Chest radiograph may shows alveolar patchy or lobar infiltration and cavity like lesion. These findings are similar to our patient. Treatment of choice is trimethoprim $15 \mathrm{mg} / \mathrm{kg} /$ day. Trimethoprime is effective for treating both nocardiosis and PJP. However, other antibiotics, for instance, imipenem and aminoglycosides plays roles in severe pulmonary nocardiosis. [7]. Brain computed tomography (CT) is essential for evaluating complcated cerebral abscess in nocardiosis [8]. Cutaneous nocardiosis abscess concomitant with PJP has been reported in patient receiving systemic corticosteroid [9]. Baucase of the protective immunity response to both Nocardia and PJP is primarily a T-cell mediated mechanism [10] [11]. Despite that of chest CT scan has the advantages in diagnosis pulmonary infection and has been widely utilized. The diffuse ground-glass opacities in chest CT is suggestive but nonspecific for PJP and CMV in immunocompromised patients [12]. For this reason, the pathogen confirmation is mandatory for diagnosis and treatment. Although gold standard for definitive diagnosis of CMV pneumonitis is cytopathic change in cytopathology of respiratory speciments [13]. The treatment for probable CMV pneumonitis is considered in high-risk patients with high BALF CMV viral load respresenting CMV organ diseases [14]. The polymicrobial infections are common in aggressively immunosuppressed hosts and in the absence of antimicrobial prophylaxis. The clinical recognition and vigourius treatment for the concomitant pathogens are mutually exclusive in the patients with delayed improvement or clinical worsening.

\section{Conclusion}

Multiple respiratory organisms are frequently encountered in pneumonia which is not common, however it is possible in immunocompromised patients. After treatment of the initially detected organism, reevaluation should be performed in the worsening of clinical presentation. Since PJP is a common infectious pulmonary complication in solid organ transplantations and it is associated with high morbidity and mortality. The prophylaxis administration is recommended in the specific immunocompromised group. Coinfected PJP and nocardiosis is not rare. The defective $\mathrm{T}$ cell mediated immunity response for both pathogens. Since the role CMV viral load in BALF and in serum for diagnosis of CMV pneu- 
monitis remains controversy. However, the high level of viral in BALF load should be concern for the probable CMV pneumonitis treatment in the absence of cytopathological confirmation.

\section{Conflicts of Interest}

The authors declare no conflicts of interest regarding the publication of this paper.

\section{References}

[1] Rosenow, E.C., Wilson, W.R. and Cockerill, F.R. (1985) Pulmonary Disease in the Immunocompromised Host. Mayo Clinic Proceedings, 60, 473-487. https://doi.org/10.1016/S0025-6196(12)60872-6

[2] Sethi, S. (2009) Pneumonia in Immunocompromised Patients. Respirology, 14, S44-50. https://doi.org/10.1111/j.1440-1843.2009.01576.x

[3] Peck, K.R., Kim, T.J., Lee, M.A., Lee, K.S. and Han, J. (2018) Pneumonia in Immunocompromised Patients: Updates in Clinical and Imaging Features. Precision and Future Medicine, 2, 95-108. https://doi.org/10.23838/pfm.2018.00121

[4] White, P.L., Backx, M. and Barnes, R.A. (2017) Diagnosis and Management of Pneumocystis jirovecii Infection. Expert Review of Anti-Infective Therapy, 15, 435-447. https://doi.org/10.1080/14787210.2017.1305887

[5] Salzer, H.J.F., Schäfer, G., Hoenigl, M., Günther, G., Hoffmann, C., Kalsdorf, B, et al. (2018) Clinical, Diagnostic, and Treatment Disparities between HIV-Infected and Non-HIV-Infected Immunocompromised Patients with Pneumocystis jirovecii Pneumonia. Respiration, 96, 52-65. https://doi.org/10.1159/000487713

[6] Nahimana, A., Rabodonirina, M., Bille, J., Francioli, P. and Hauser, P.M. (2004) Mutations of Pneumocystis jirovecii Dihydrofolate Reductase Associated with Failure of Prophylaxis. Antimicrobial Agents and Chemotheraphy, 48, 4301-5. https://doi.org/10.1128/AAC.48.11.4301-4305.2004

[7] Restrepo, A. and Clark, N.M. (2019) Transplantation obotIDCoPotASo. Nocardia Infections in Solid Organ Transplantation: Guidelines from the Infectious Diseases Community of Practice of the American Society of Transplantation. The Journal of Clinical and Translational Research, 33, e13509. https://doi.org/10.1111/ctr.13509

[8] Mootsikapun, P., Intarapokam, B. and Liawnoraset, W. (2005) Nocardiosis in Srinagarind Hospital, Thailand: Review of 70 Cases from 1996-2001. International Journal of Infectious Diseases, 9, 154-158. https://doi.org/10.1016/j.ijid.2004.06.011

[9] Verstockt, B., Van Hemelen, M., Outtier, A., Sabino, J., Van Wijngaerden, E., De Munter, P., et al. (2020) Invasive Nocardiosis, Disseminated Varicella Zoster Reactivation, and Pneumocystis jiroveci Pneumonia Associated with Tofacitinib and Concomitant Systemic Corticosteroid Use in Ulcerative Colitis. Journal of Gastroenterology and Hepatology, 35, 2294-2297. https://doi.org/10.1111/igh.15256

[10] Deem, R.L., Doughty, F.A. and Beaman, B.L. (1983) Immunologically Specific Direct $\mathrm{T}$ Lymphocyte-Mediated Killing of Nocardia Asteroides. The Journal of Immunology, 130, 2401-2406.

[11] Shellito, J., Suzara, V.V., Blumenfeld, W., Beck, J.M., Steger, H.J. and Ermak, T.H. (1990) A New Model of Pneumocystis Carinii Infection in Mice Selectively Depleted of Helper T Lymphocytes. Journal of Clinical Investigation, 85, 1686-1693. https://doi.org/10.1172/JCI114621 
[12] Du, C.-J., Liu, J.-Y., Chen, H., Yan, S., Pu, L., Xiong, H.-F., et al. (2020) Differences and Similarities of High-Resolution Computed Tomography Features between Pneumocystis Pneumonia and Cytomegalovirus Pneumonia in AIDS Patients. Infectious Diseases of Poverty, 9, 149. https://doi.org/10.1186/s40249-020-00768-2

[13] Ljungman, P., Boeckh, M., Hirsch, H.H., Josephson, F., Lundgren, J., Nichols, G., et al. (2017) Definitions of Cytomegalovirus Infection and Disease in Transplant Patients for Use in Clinical Trials. Clinical Infectious Diseases, 64, 87-91.

https://doi.org/10.1093/cid/ciw668

[14] Lee, H.Y., Rhee, C.K., Choi, J.Y., Lee, H.Y., Lee, J.W. and Lee, D.G. (2017) Diagnosis of Cytomegalovirus Pneumonia by Quantitative Polymerase Chain Reaction Using Bronchial Washing Fluid from Patients with Hematologic Malignancies. Oncotarget, 8, 39736-39745. https://doi.org/10.18632/oncotarget.14504 